\title{
The molluscs and their habitats in Sashtinska Sredna Gora Mts. (Southern Bulgaria)
}

\author{
Dilian Georgiev Georgiev ${ }^{1} \&$ Slaveya Borisova Stoycheva ${ }^{2}$ \\ ${ }^{I}$ Department of Ecology and Environmental Conservation, Faculty of Biology, University of Plovdiv, Tzar Assen Str. 24, BG-4000 \\ Plovdiv, Bulgaria, e-mail: diliangeorgiev@abv.bg \\ ${ }^{2}$ NGO Green Balkans, Shesti septemvri Str. 160, BG-4000 Plovdiv, Bulgaria, e-mail: slaveyastoycheva@abv.bg
}

Georgiev D.G. \& Stoycheva S.B., 2009: The molluscs and their habitats in Sashtinska Sredna Gora Mts. (Southern Bulgaria). - Malacologica Bohemoslovaca, 8: 1-8. Online serial at $<$ http://mollusca.sav.sk $>$ 28-Jan-2009.

\begin{abstract}
The aim of this study was to investigate the mollusc species diversity of Sashtinska Sredna Gora Mts. (Southern Bulgaria) in detail, and to obtain some data on the distribution of the malacofauna both in habitats and in geographical areas of this mountain. The survey was carried out during the period of 20 Oct 2007 - 12 Nov 2008. Total 49 localities were examined, and their co-ordinates were given, 78 mollusc species were registered in the study area, from which 75 were new records for Sashtinska Sredna Gora Mts. Seventeen molluscan habitats were studied separately, from which the forests on river banks dominated by Salix sp. and Alnus glutinosa, open grassy terrains with short vegetation, and oak forests showed the highest species diversity. Considering the base rock type molluscan communities were separated in two main groups: a complex of species in the limestone areas, and another one inhabiting the volcanic/non limestone terrains. The artificial substrate even considered as a calcium source, in our area had played a little role on diversity. It held a species complex not so close related to the limestone faunas than with the volcanic base rock ones, on which it was situated.
\end{abstract}

Key words: Gastropoda, Bivalvia, diversity, rocks, limestone, volcanic, Balkans

\section{Introduction}

There was insufficient information on the malacofauna of one not so high mountain of Bulgaria - the Sashtinska Sredna Gora Mts. (maximal height of $1604 \mathrm{~m}$ alt.) (Hubenov, 2005). Only a few papers were published and a number of species recorded. DAmJAnov \& LikHAREV (1975) reported Pomatias elegans (O.F. Müller 1774) for the "central part of Sredna Gora Mountain" (which possibly means Sashtinska Sredna Gora Mts., see "Material and Methods"), and Bulgarica denticulata (Olivier 1801) near Bogdan Peak. WIKTOR (1983) found Lehmannia nyctelia (Bourguignat 1855) at Koprivshtitza town, Deroceras bureschi (H. Wagner 1934) near Bogdan Hut, and Arion subfuscus (Draparnaud 1801) in both localities mentioned. Recently Georgiev \& Stoycheva (2008) registered the freshwater Bythinella opaca (Gallenstein 1848) near the village of Dijulevo.

The aim of our study was to investigate the species diversity of the Sashtinska Sredna Gora Mts. in detail, and to obtain some data on the distribution of the malacofauna both in habitats and in geographical areas of this mountain.

\section{Material and Methods}

Sashtinska Sredna Gora Mts. are situated in the south of the country (Fig. 1), bordering with the Stara Planina Mts. in the north and with the Thracian Lowland in the south. To the west and east it is close to the Ichtimanska and Sarnena Sredna Gora Mountains, respectively, as these three mountains are parts of one large massif named the Sredna Gora Mts. This ridge is divided on its three parts by two rivers: Topolnitza (between the Ichtimanska and Sashtinska Sredna Gora) and Stryama (between the Sashtinska and Sarnena Gora). Sashtinska Sredna Gora Mts. is the highest one from the three massifs, with its highest point - Bogdan Peak (1604 $\mathrm{m}$ a.s.1.), and it has the most expressive and steep ridge. The mountain is consisted mainly by volcanic rocks, but a few limestone "islands" also present at its central and north-eastern parts. The forests are dominated mainly by Quercus spp. in the low hills and Fagus sylvatica in the high parts of the massif. The human population of the mountain is not big and is located in small villages and few towns.

The survey was carried out during the period of 20 Oct 2007 - 12 Nov 2008. Material was gathered from 49 localities in the mountain (Fig. 1, Table 1). All molluscs were collected by the authors, and were studied by means of the standard procedures (KERNEY et al. 1983). The material collected was identified following ZHADIN (1952), DAMJANOV \& LiKhAREV (1975), KeRney et al. (1983), WikTOR (1983), Hausdorf (2000), GlÖEr \& Meier-Brook (2003). The following mollusc habitats were considered for this study (Table 2): Freshwater habitats: streams (st), termal springs (ts), canals (can), medium sized rivers (riv), standing waters as small ponds and micro dams (sw), Land habitats: river bank forests dominated by Salix sp. and Alnus glutinosa, sometimes with Ulmus sp. and Juglans regia (Sf), littoral vegetation on water basins banks as Typha sp. and Phragmytes australis (lv), short grass vegetation 


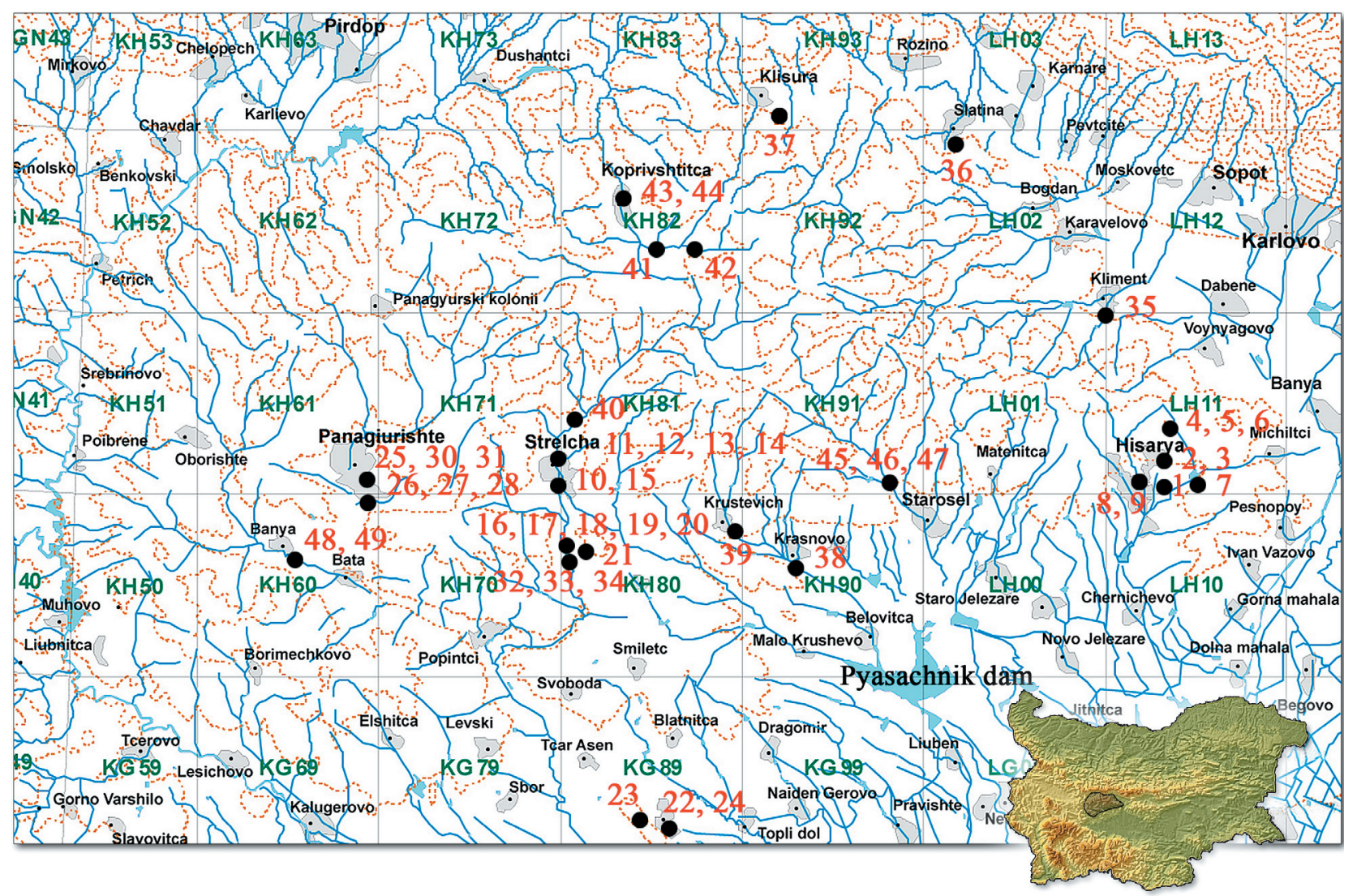

Fig. 1. Study Area - position of the Sashtinska Srenda Gora Mts. and the localities examined for mollusk species.

(shg), tall grass vegetation as Urtica sp., Mentha sp. and others (tg), agricultural lands (al), bush vegetation dominated mainly by Paliurus spina-christii, Rosa sp. and Prunus spinosa (bv), broad leaf xeric forests dominated by Quercus sp., sometimes with sub-dominant Carpinus orientalis (Qf), Carpinus betulus forest (Cf), Fagus sylvatica forests, sometimes with sub-dominant Carpinus betulus (Ff), park forests in urbanized areas (pf), house yards (hy), unknown habitat: specimens found only in river deposits (rd).

Sorensen qualitative similarity index (S) was evaluated to compare the faunas of different sites (DAJO, 1975). Cluster analysis was used to investigate the relationship between the mollusc communities on different types of base rock terrains in the area under study by computer program Statistica for Windows 7.0 by unweighted pair group average qualitative measure.

\section{Results and Discussion}

A total of 78 mollusc species were registered in the study area, from which 75 were new records for the Sashtinska Sredna Gora Mts. (Table 2). We did not find the species reported from the mountain as: Pomatias elegans, Bulgarica denticulata, and Deroceras bureschi. Summing these species with those collected in present study, we found that till now 81 species of molluscs are known for this mountain. A few freshwater species we found at the near by pre-mountains south of the study area (but not in the mountain), in Pyasachnik Dam (UTM-grid LG09, see Fig. 1), could possibly also be expected and in the higher terrains: Viviparus acerosus (Bourguignat, 1862), Radix auricularia (Linnaeus, 1758), and Dreissena polymorpha

\section{(Pallas, 1771).}

For comparison at the nearby rich on limestone the Sarnena Sredna Gora Mts., Georgiev \& Georgiev (2002, 2003, 2004), and Georgiev (2003, 2005) found a total of 5 species of Bivalvia, 12 freshwater and 67 land species of gastropods both in urban and natural habitats (total 84 species of molluscs). After these studies we found and another three new taxa for this mountain: Vertigo antivertigo (Draparnaud, 1801) (27 Oct 2007, deposits of the Bedechka River, north of the Stara Zagora town), Chondrina avenacea (Bruguiere, 1792) (22 Mar 2008, north of the Novo Selo village), and Anodonta anatina (Linnaeus, 1758) (15 Nov 2008, a pond in the Starozagorski Bani resort). So, for now 87 species of molluscs are known for the Sarnena Gora Mts., with 6 more than in the Sashtinska Sredna Gora Mts. found during present study. The Sorensen qualitative measure showed $69 \%$ of similarity between the faunas in the two mountains discussed.

The richest diversity of mollusc species in our study area held the forests on the river banks dominated by Salix sp. and Alnus glutinosa $(\mathrm{n}=24 \mathrm{sp}$., 30.8\% from all registered), open grassy terrains with short vegetation $(\mathrm{n}=$ 22 sp., $28.2 \%$ ), and the oak forests ( $\mathrm{n}=20 \mathrm{sp}$., $25.6 \%)$. The other habitats were very poor on mollusc taxa having a diversity ranging between 2 and 14 species. From the freshwater habitats we found that richest were the medium sized rivers in the mountain with 9 species (snails and mussels).

As a whole the results showed that habitats of the Sashtinska Sredna Gora Mts. were relatively poor on mollusc species comparing with data of GEORGIEV (2005) for the large limestone areas of the Sarnena Sredna Gora Mts. 
Table 1. Localities of gathering the mollusks from Sashtinska Sredna Gora Mountain. Abbreviations: loc. = locality, UTM-grid = UTM-grid 10x10 km, alt. = altitude, $\mathrm{nm}=$ not measured

\begin{tabular}{|c|c|c|c|c|c|}
\hline loc. & date & landmark & UTM-grid & GPS co-ordinates & alt. \\
\hline 1 & 20 Oct 2007 & Hisarya town, residential area of Miromir & LH 10 & $42^{\circ} 29^{\prime} 17.9^{\prime \prime} \mathrm{N}, 24^{\circ} 42^{\prime} 56.3^{\prime \prime} \mathrm{E}$ & 282 \\
\hline 2 & 20 Oct 2007 & north-east of Hisarya town & LH 11 & $42^{\circ} 2934.0^{\prime \prime} \mathrm{N}, 24^{\circ} 43^{\prime} 03.7^{\prime \prime} \mathrm{E}$ & 282 \\
\hline 3 & 20 Oct 2007 & north of locality №2 & LH 11 & $\mathrm{~nm}$ & $\mathrm{~nm}$ \\
\hline 4 & 20 Oct 2007 & north-east of Hisarya town & LH 11 & $42^{\circ} 30^{\prime} 48.8^{\prime \prime} \mathrm{N}, 24^{\circ} 43^{\prime} 59.4^{\prime \prime} \mathrm{E}$ & 336 \\
\hline 5 & 21 Oct 2007 & north-east of Hisarya town & LH 11 & $42^{\circ} 31^{\prime} 10.5^{\prime \prime} \mathrm{N}, 24^{\circ} 43^{\prime} 59.4^{\prime \prime} \mathrm{E}$ & 388 \\
\hline 6 & 21 Oct 2007 & Hisarya town - northern part & LH 11 & $\mathrm{~nm}$ & $\mathrm{~nm}$ \\
\hline 7 & 21 Oct 2007 & south-east of Hisarya town & LH 11 & $\mathrm{~nm}$ & $\mathrm{~nm}$ \\
\hline 8 & 21 Oct 2007 & central part of Hisarya town & LH 10 & $42^{\circ} 29^{\prime} 49.7^{\prime \prime} \mathrm{N}, 24^{\circ} 42^{\prime} 24.8^{\prime \prime} \mathrm{E}$ & 349 \\
\hline 9 & 21 Oct 2007 & near the railway station of Hisarya town & LH 10 & $42^{\circ} 30^{\prime} 19.8^{\prime \prime} \mathrm{N}, 24^{\circ} 42^{\prime} 05.1^{\prime \prime} \mathrm{E}$ & 370 \\
\hline 10 & 03 Nov 2007 & Streltcha town, near Streltchenska Luda Yana River & KH 80 & $42^{\circ} 30^{\prime} 02.2^{\prime \prime} \mathrm{N}, 24^{\circ} 19^{\prime} 29.5^{\prime \prime} \mathrm{E}$ & 431 \\
\hline 11 & 03 Nov 2007 & north of Streltcha town, near Streltchenska Luda Yana River & KH 81 & $\mathrm{~nm}$ & $\mathrm{~nm}$ \\
\hline 12 & 03 Nov 2007 & north of Streltcha town & KH 81 & $42^{\circ} 31^{\prime} 20.1^{\prime \prime} \mathrm{N}, 24^{\circ} 19^{\prime} 30.9^{\prime \prime} \mathrm{E}$ & 415 \\
\hline 13 & 03 Nov 2007 & north of Streltcha town & KH 81 & $42^{\circ} 31^{\prime} 56.9^{\prime \prime} \mathrm{N}, 24^{\circ} 19^{\prime} 51.5^{\prime \prime} \mathrm{E}$ & 470 \\
\hline 14 & 03 Nov 2007 & Streltcha town, west tributary of Streltchenska Luda Yana River & KH 80 & $42^{\circ} 31^{\prime} 23.1^{\prime \prime} \mathrm{N}, 24^{\circ} 19^{\prime} 17.0^{\prime \prime} \mathrm{E}$ & 422 \\
\hline 15 & 03 Nov 2007 & near the railway station of Streltcha town & KH 80 & $42^{\circ} 29^{\prime} 34.0^{\prime \prime} \mathrm{N}, 24^{\circ} 19^{\prime} 53.0^{\prime \prime} \mathrm{E}$ & 399 \\
\hline 16 & 24 Nov 2007 & west of the railway station of Dijulevo village & KH 80 & $42^{\circ} 27^{\prime} 07.2^{\prime \prime} \mathrm{N}, 24^{\circ} 21^{\prime} 58.6^{\prime \prime} \mathrm{E}$ & 472 \\
\hline 17 & 24 Nov 2007 & a small pond west of Dijulevo village & KH 80 & $42^{\circ} 27^{\prime} 13.3^{\prime \prime} \mathrm{N}, 24^{\circ} 20^{\prime} 34.1^{\prime \prime} \mathrm{E}$ & 422 \\
\hline 18 & 24 Nov 2007 & a small pond west of Dijulevo village & KH 80 & $42^{\circ} 27^{\prime} 15.6^{\prime \prime} \mathrm{N}, 24^{\circ} 20^{\prime} 27.1^{\prime \prime} \mathrm{E}$ & 393 \\
\hline 19 & 24 Nov 2007 & west of Dijulevo village, a tributary of Luda Yana River & KH 80 & $42^{\circ} 27^{\prime} 13.5^{\prime \prime} \mathrm{N}, 24^{\circ} 20^{\prime} 18.7^{\prime \prime} \mathrm{E}$ & 362 \\
\hline 20 & 24 Nov 2007 & west of Dijulevo village, Luda Yana River & KH 80 & $42^{\circ} 26^{\prime} 55.5^{\prime \prime} \mathrm{N}, 24^{\circ} 20^{\prime} 02.1^{\prime \prime} \mathrm{E}$ & 325 \\
\hline 21 & 24 Nov 2007 & railway station of Dijulevo village & KH 80 & $\mathrm{~nm}$ & $\mathrm{~nm}$ \\
\hline 22 & 08 Mar 2008 & railway station of Ovchepoltzi village & KG89 & $\mathrm{nm}$ & $\mathrm{nm}$ \\
\hline 23 & 08 Mar 2008 & west of railway station of Ovchepoltzi village & KG89 & $42^{\circ} 20^{\prime} 21.5^{\prime \prime} \mathrm{N}, 24^{\circ} 22^{\prime} 34.1^{\prime \prime} \mathrm{E}$ & 500 \\
\hline 24 & 08 Mar 2008 & east of railway station of Ovchepoltzi village & KG89 & $\mathrm{nm}$ & $\mathrm{nm}$ \\
\hline 25 & 29 Mar 2008 & Panagyurishte town & KH60 & $42^{\circ} 29^{\prime} 21.5^{\prime \prime} \mathrm{N}, 24^{\circ} 11^{\prime} 52.2^{\prime \prime} \mathrm{E}$ & 475 \\
\hline 26 & 29 Mar 2008 & south of Panagyurishte town & KH60 & $42^{\circ} 29^{\prime} 15.2^{\prime \prime} \mathrm{N}, 24^{\circ} 11^{\prime} 47.8^{\prime \prime} \mathrm{E}$ & 478 \\
\hline 27 & 29 Mar 2008 & south of Panagyurishte town & KH60 & $42^{\circ} 29^{\prime} 12.1^{\prime \prime} \mathrm{N}, 24^{\circ} 11^{\prime} 35.5^{\prime \prime} \mathrm{E}$ & 476 \\
\hline 28 & 29 Mar 2008 & south of Panagyurishte town, Panagyurska Luda Yana River & KH60 & $42^{\circ} 29^{\prime} 02.4^{\prime \prime} \mathrm{N}, 24^{\circ} 11^{\prime} 23.5^{\prime \prime} \mathrm{E}$ & 478 \\
\hline 29 & 29 Mar 2008 & south of locality №28 & KH60 & $42^{\circ} 28^{\prime} 44.5^{\prime \prime} \mathrm{N}, 24^{\circ} 11^{\prime} 10.4^{\prime \prime} \mathrm{E}$ & 481 \\
\hline 30 & 29 Mar 2008 & near the railway station of Panagyurishte town & KH60 & $42^{\circ} 29^{\prime} 28.9^{\prime \prime} \mathrm{N}, 24^{\circ} 11^{\prime} 52.3^{\prime \prime} \mathrm{E}$ & 470 \\
\hline 31 & 29 Mar 2008 & near the railway station of Panagyurishte town & KH60 & $42^{\circ} 29^{\prime} 30.4^{\prime \prime} \mathrm{N}, 24^{\circ} 11^{\prime} 53.1^{\prime \prime} \mathrm{E}$ & 471 \\
\hline 32 & 17 Apr 2008 & west of Dijulevo village & KH 80 & $42^{\circ} 27^{\prime} 16.1^{\prime \prime} \mathrm{N}, 24^{\circ} 21^{\prime} 14.3^{\prime \prime} \mathrm{E}$ & 558 \\
\hline 33 & 17 Apr 2008 & west of Dijulevo village & KH 80 & $42^{\circ} 27^{\prime} 00.5^{\prime \prime} \mathrm{N}, 24^{\circ} 21^{\prime} 46.6^{\prime \prime} \mathrm{E}$ & 521 \\
\hline 34 & 17 Apr 2008 & south of Dijulevo village & KH 80 & $\mathrm{~nm}$ & $\mathrm{~nm}$ \\
\hline 35 & 16 Aug 2008 & village of Kliment & LH01 & $42^{\circ} 36^{\prime} 06.6^{\prime \prime} \mathrm{N}, 24^{\circ} 41^{\prime} 19.5^{\prime \prime} \mathrm{E}$ & 382 \\
\hline 36 & 16 Aug 2008 & near village of Slatina & LH02 & $42^{\circ} 41^{\prime} 03.6^{\prime \prime} \mathrm{N}, 24^{\circ} 35^{\prime} 19.1^{\prime \prime} \mathrm{E}$ & 515 \\
\hline 37 & 16 Aug 2008 & east of Klisura town & KH92 & $42^{\circ} 41^{\prime} 58.1^{\prime \prime} \mathrm{N}, 24^{\circ} 30^{\prime} 41.5^{\prime \prime} \mathrm{E}$ & 588 \\
\hline 38 & 18 Oct 2008 & south of Krasnovo village & KH90 & $42^{\circ} 27^{\prime} 32.5^{\prime \prime} \mathrm{N}, 24^{\circ} 29^{\prime} 24.5^{\prime \prime} \mathrm{E}$ & 325 \\
\hline 39 & 18 Oct 2008 & village of Krastevitch & KH80 & $42^{\circ} 28^{\prime} 45.5^{\prime \prime} \mathrm{N}, 24^{\circ} 26^{\prime} 26.3^{\prime \prime} \mathrm{E}$ & 341 \\
\hline 40 & 18 Oct 2008 & north of Streltcha town & KH81 & $42^{\circ} 34^{\prime} 35.2^{\prime \prime} \mathrm{N}, 24^{\circ} 21^{\prime} 01.1^{\prime \prime} \mathrm{E}$ & 954 \\
\hline 41 & 18 Oct 2008 & near the road to Barikadite Hut & KH82 & $42^{\circ} 36^{\prime} 11.3^{\prime \prime} \mathrm{N}, 24^{\circ} 23^{\prime} 32.0^{\prime \prime} \mathrm{E}$ & 996 \\
\hline 42 & 18 Oct 2008 & east of locality №41 & KH82 & $42^{\circ} 35^{\prime} 20.9^{\prime \prime} \mathrm{N}, 24^{\circ} 24^{\prime} 49.4^{\prime \prime} \mathrm{E}$ & 979 \\
\hline 43 & 18 Oct 2008 & central part of Koprivshtitza town & KH82 & $42^{\circ} 38^{\prime} 17.4^{\prime \prime} \mathrm{N}, 24^{\circ} 21^{\prime} 40.0^{\prime \prime} \mathrm{E}$ & 935 \\
\hline 44 & 18 Oct 2008 & north-east part of Koprivstitza town & KH82 & $42^{\circ} 38^{\prime} 27.9^{\prime \prime} \mathrm{N}, 24^{\circ} 21^{\prime} 45.4^{\prime \prime} \mathrm{E}$ & 940 \\
\hline 45 & 5 Nov 2008 & north of Starosel village, near Pyasachnik river & KH91 & $42^{\circ} 30^{\prime} 39.2^{\prime \prime} \mathrm{N}, 24^{\circ} 32^{\prime} 23.6^{\prime \prime} \mathrm{E}$ & 344 \\
\hline 46 & 5 Nov 2008 & north of Starosel village, beneath the Manev Dol dam`s wall & KH91 & $42^{\circ} 30^{\prime} 35.5^{\prime \prime} \mathrm{N}, 24^{\circ} 32^{\prime} 21.1^{\prime \prime} \mathrm{E}$ & 351 \\
\hline 47 & 5 Nov 2008 & Manev Dol dam & KH91 & $42^{\circ} 30^{\prime} 19.3^{\prime \prime} \mathrm{N}, 24^{\circ} 32^{\prime} 4.6^{\prime \prime} \mathrm{E}$ & 363 \\
\hline 48 & 12 Nov 2008 & east of Banja village & KH60 & $42^{\circ} 27^{\prime} 26.1^{\prime \prime} \mathrm{N}, 24^{\circ} 09^{\prime} 31.1^{\prime \prime} \mathrm{E}$ & 454 \\
\hline 49 & 12 Nov 2008 & north of locality №48 & KH60 & $\mathrm{nm}$ & $\mathrm{nm}$ \\
\hline
\end{tabular}




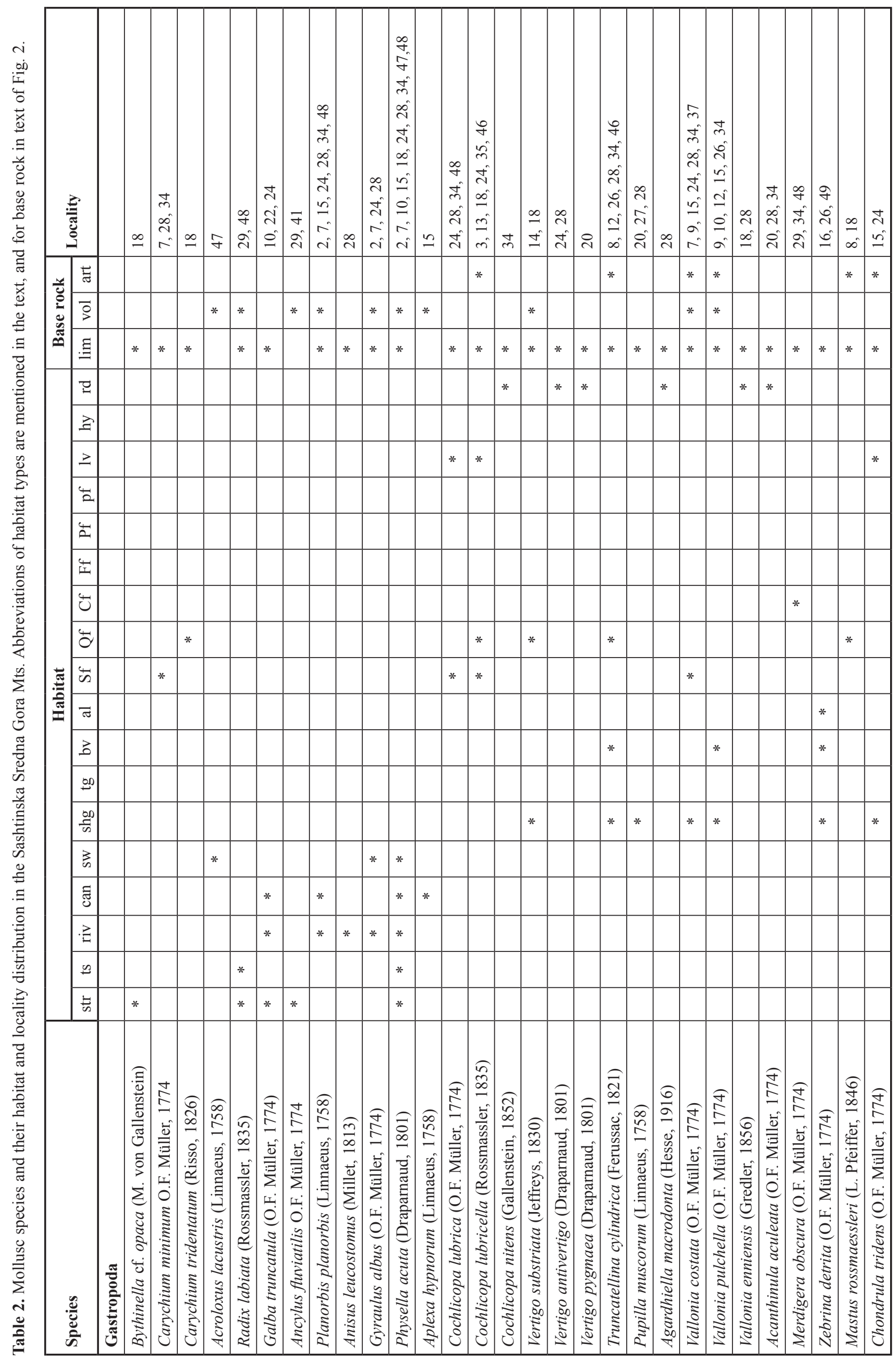




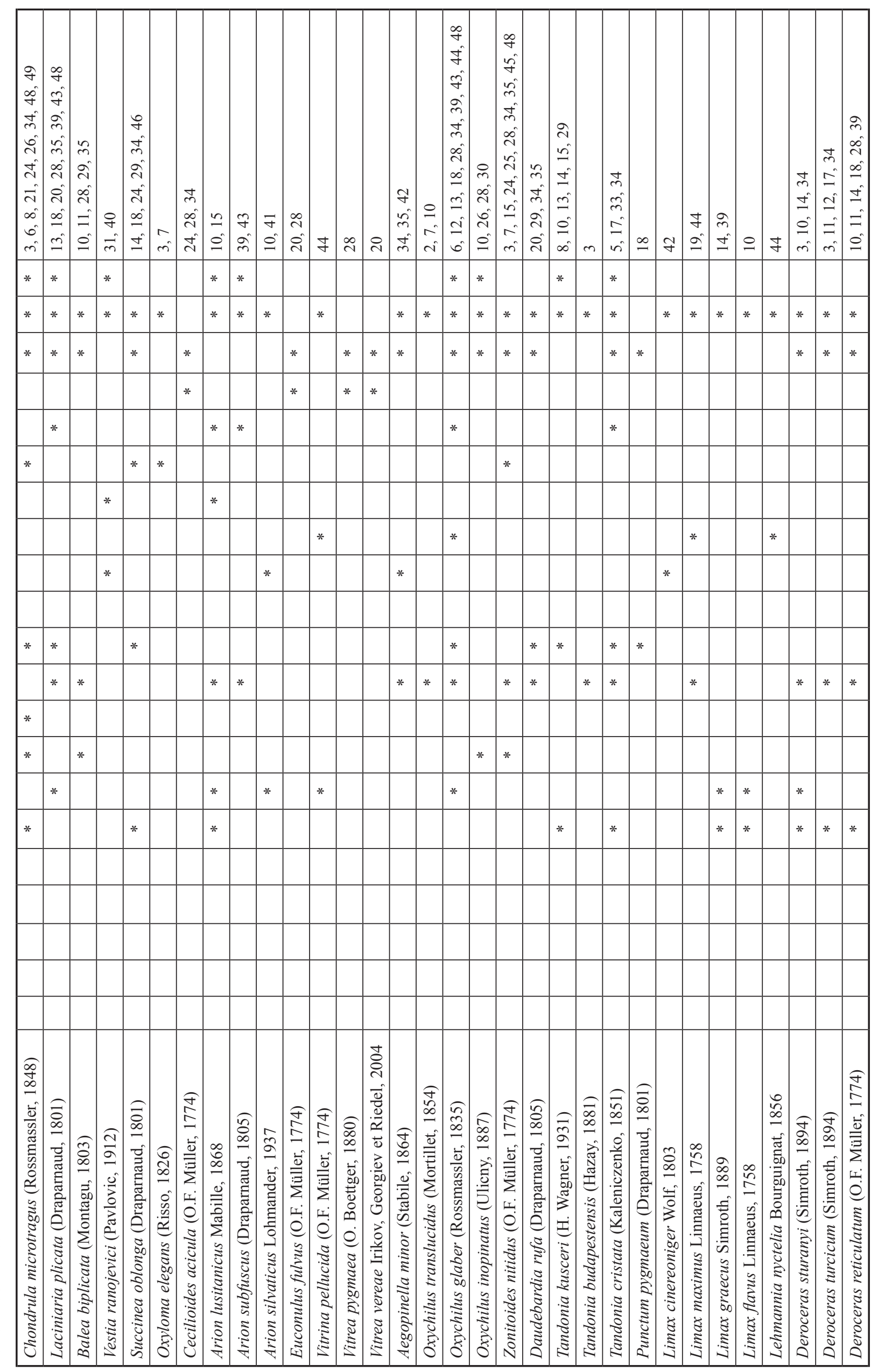




\begin{tabular}{|c|c|c|c|c|c|c|c|c|c|c|c|c|c|c|c|c|c|c|c|}
\hline F & 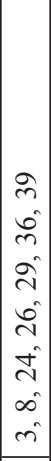 & $\begin{array}{l}\hat{y} \\
\hat{\jmath} \\
\hat{\jmath} \\
\infty \\
\infty \\
\dot{v}\end{array}$ & 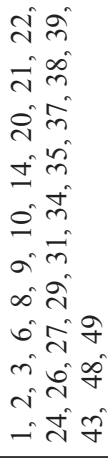 & $\begin{array}{l}\hat{n} \\
\hat{i} \\
\infty \\
i \\
0 \\
\hat{n} \\
\hat{n} \\
\hat{n}\end{array}$ & 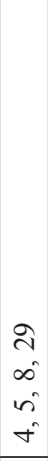 & 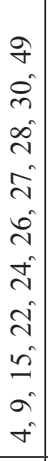 & 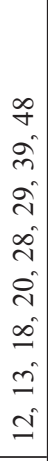 & \begin{tabular}{|l} 
\\
\\
\\
\\
\\
\\
0 \\
0 \\
0 \\
0
\end{tabular} & 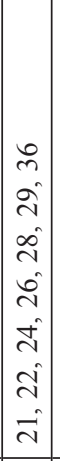 & $\begin{array}{l}2 \\
0 \\
0 \\
m\end{array}$ & 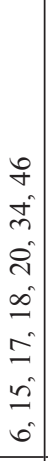 & 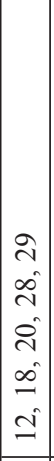 & & $\stackrel{む}{\Delta}$ & $r$ & $\begin{array}{c} \pm \\
m \\
\infty \\
i \\
\infty \\
\infty\end{array}$ & $\stackrel{\infty}{\sim}$ & $\stackrel{\infty}{i}$ & \\
\hline & * & $*$ & * & $*$ & $*$ & * & $*$ & $*$ & $*$ & $*$ & * & & & & & & & & i \\
\hline * & * & $*$ & * & & * & * & 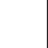 & & * & * & * & & & & * & & & & 字 \\
\hline & * & $*$ & * & * & * & * & * & & * & * & * & * & & * & & * & * & * & $\infty$ \\
\hline & & & & & & & & & & & & & & & & & & & 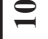 \\
\hline & & & * & & $*$ & & & $*$ & & & & & & & & & & & $\infty$ \\
\hline & * & & $*$ & & & $*$ & & & $*$ & & & & & & & & & & $\varrho$ \\
\hline & * & $*$ & * & & & & & * & & & & & & & & & & & 6 \\
\hline & & & & & & & & & & & & & & & & & & & $\nabla$ \\
\hline * & & & & & & & & & & & & & & & & & & & in \\
\hline & * & & $*$ & * & * & & $*$ & & & & & * & & & & & & & $n$ \\
\hline & * & & $*$ & $*$ & $*$ & & $*$ & & & & * & $*$ & & & & & & & 로 \\
\hline & * & & * & $*$ & & & $*$ & & & & & $*$ & & & & & & & $\stackrel{\sim}{\sim}$ \\
\hline & & & * & & & * & & & & & & & & & & & & & $\nabla$ \\
\hline & * & $*$ & * & * & * & * & & & * & * & & & & & & & & & \pm \\
\hline & * & & * & & & & * & & * & & & & & & & & & & $=$ \\
\hline & & & * & & & * & & $*$ & $*$ & $*$ & * & & & & & & & & $\approx$ \\
\hline & & & & & & & & & & & & & & & & & & & $m$ \\
\hline & & & & & & & & & & & & & & & & & & & I \\
\hline & & & & & & & & & & & & & & $*$ & $*$ & $*$ & & $*$ & a \\
\hline & & & & & & & & & & & & & & & & & & & $N$ \\
\hline & & & & & & & & & & & & & & & & $*$ & $*$ & & 1 \\
\hline 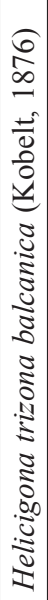 & 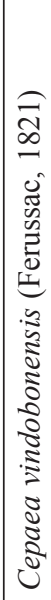 & 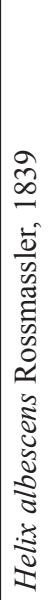 & 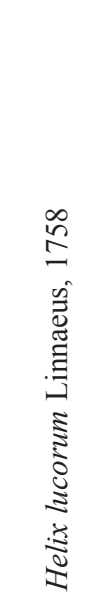 & 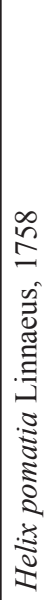 & 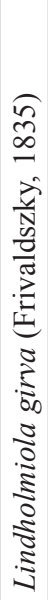 & 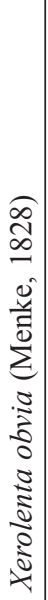 & 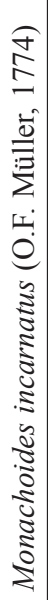 & 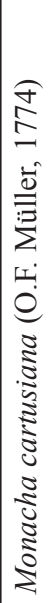 & 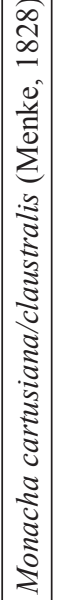 & 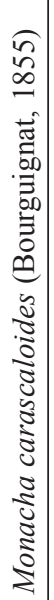 & 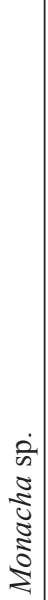 & 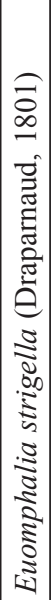 & $\mid$ & 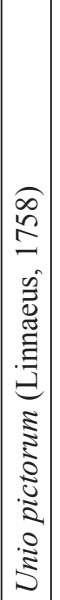 & 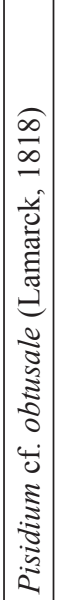 & 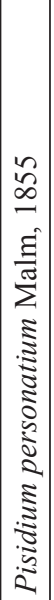 & 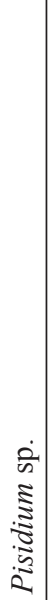 & 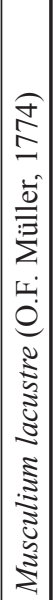 & 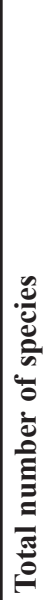 \\
\hline
\end{tabular}




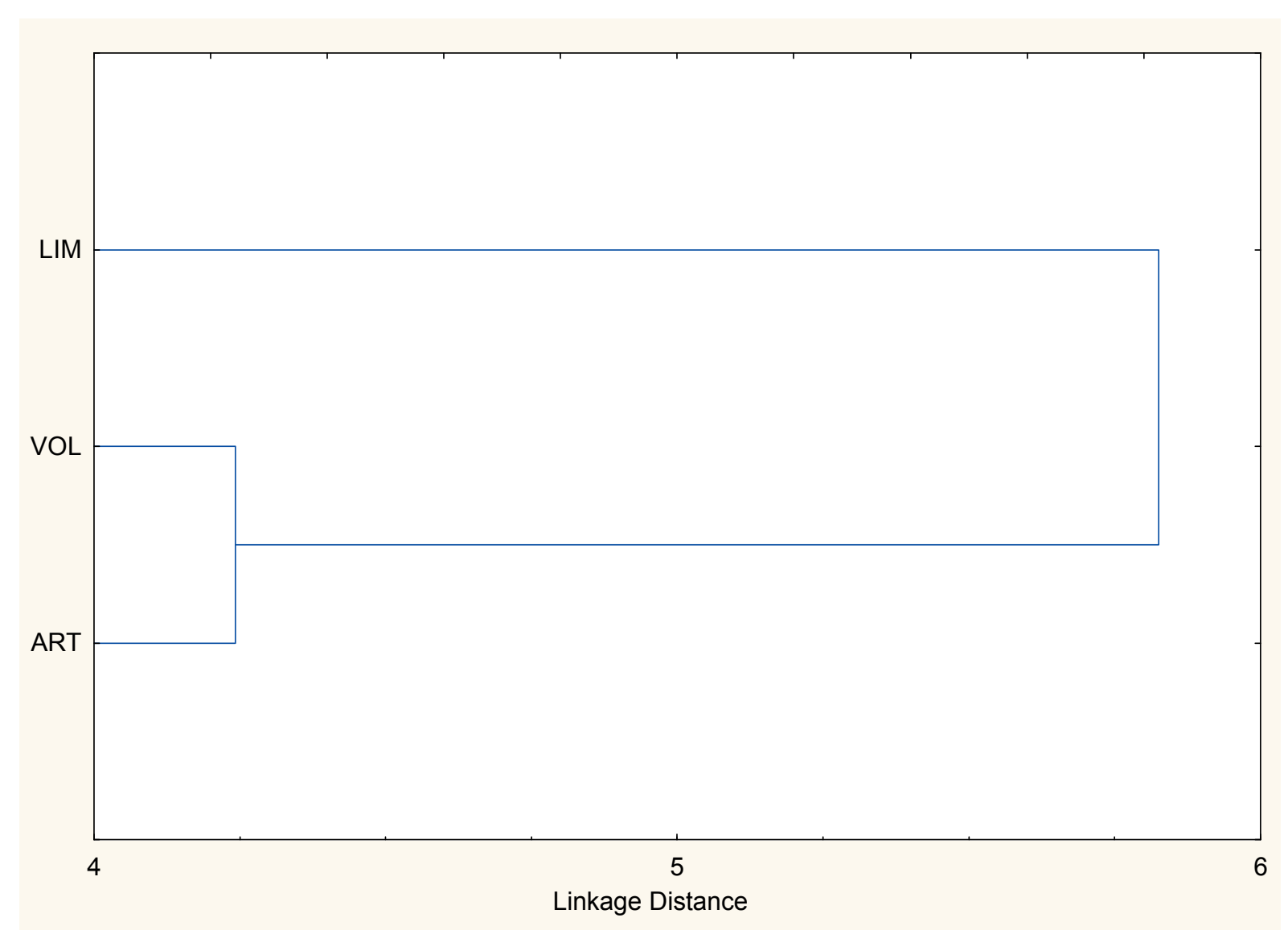

Fig. 2. Cluster analysis used to examine the relationship between mollusk communities placed on different base rock types. Abbreviations: LIM - limestone, VOL - volcanic or non limestone, ART - artificial.

neighboring. In the last one for example at river site forests 53 species were found, in bush areas 41 species, oak forests 37 , coniferous forests 22 , and in the same sized rivers 17 mollusc taxa. No species in the Sashtinska Sredna Gora Mts. was registered in over $50 \%$ of the localities examined, even the widely distributed Helix lucorum (24 localities, 49\% from all). As many authors correlated the distribution of land molluscs with the presence of calcerous substrates (LožEK 1962, ANT 1963), it was evident that despite the similar number of species recorded in both neighboring mountains, in our area the molluscs were rare and scattered for some reason. Such a comparative studies were carried out by Schilthuizen et al. (2003) for Borneo hills, and authors found that diversities on limestone were similar to those in non-limestone areas. Having such information we compared the mollusc communities in the Sashtinska Sredna Gora Mts. according to the base rock of the habitats. Knowing that some snails are using artificial (mainly building) materials as a source of calcium (LožEK 1962, Kalisz \& Powell 2003), we divided three types of "base rock": limestone, volcanic and artificial materials on volcanic terrains (concrete, mortar, bricks and other). The cluster analysis showed that in our study area the molluscan communities were separated in two main groups: a complex of species in the limestone areas, and another one inhabiting the volcanic rock terrains (Fig. 2). The artificial substrate even considered as a calcium source, in our area had played a little role. It held a species complex not so close related to the limestone faunas $(\mathrm{S}=50 \%)$ than with the volcanic base rock ones, on which it was situated ( $\mathrm{S}$
$=52.1 \%)$. The limestone and volcanic communities represented the lowest similarity $(\mathrm{S}=34.3 \%)$. It could be supposed that native calcerous mollusc species from the limestone areas did not succeed as a whole complex to invade the areas occupied by human made calcium rich structures, having restricted populations in small limestone "islands" in the mountain. Here also a complex of factors could be proposed for the possible weak species dispersion like not proper relief specifications and low levels of human transportation. Though a small group of limestone loving species did expand on terrains dominated by artificial sources of calcium as: Truncatellina cylindrica, Mastus rossmaessleri, Chondrula tridens, Chondrula microtragus, and even the last we found as few shells on volcanic rocks. Amazing was the single record of Helicigona trizona balcanica (1 live juvenile specimen) in a beech forest totally dominated by volcanic rocks. Of course in our paper we do not consider the individual abundance of all the species, which is well known that often correlates with the presence of calcium (Ноторе 2002, Schilthuizen et al. 2003), and show only its influence on species diversity in a particular area.

\section{Conclusions}

From 78 mollusc species found in the Sashtinska Sredna Gora Mts., 75 were new records for the area. As a whole the habitats were poor in species, which were restricted in localities with scattered distribution. From all habitats studied, forests on the river banks dominated by Salix sp. and Alnus glutinosa, open grassy terrains with short ve- 
getation, and the oak forests showed the highest species diversity. The malacocoenoses established on limestone terrains were not related with those on volcanic base rock. In the study area artificial substrate even considered as a calcium source had played a little role on species richness as a lot of calcerous species found on limestone sites were lacking.

\section{Acknowledgements}

We are very grateful to P. Glöer (Hetlingen, Germany) for sending us a lot of valuable literature for identification of the freshwater molluscs, for his friendly attitude, and for determination the species Pisidium personatum. We thank and to I. Velcheva and I. Mollov (Plovdiv University, Bulgaria) for their advices on preparing the manuscript.

\section{References}

ANT H., 1963: Faunistische, ökologische und tiergeographische Untersuchungen zur Verbreitung der Landschnecken in Nordwestdeutschland. - Abhandlungen aus dem Landesmuseum für Naturkunde zu Münster in Westfalen, 25(1): 125 pp.

DAJO R., 1975: Fundamentals of Ecology. - Progress Publ., Moskow, 415 pp. (in Russian).

Damjanov S. \& Likharev I., 1975: Fauna Bulgarica, 5. Terrestrial snails (Gastropoda terrestria). - Marin Drinov Publ., Sofia, 425 pp. (in Bulgarian).

Georgiev D., 2003: Land malacofauna of Stara Zagora town and its adjacent territories. - Unpublished MSc Thesis, Faculty of Biology, University of Plovdiv, 107 pp. (in Bulgarian).

Georgiev D., 2005: Species diversity and habitat distribution of the malacofauna (Mollusca: Bivalvia, Gastropoda) of Surnena Sredna Gora Mountain (Southern Bulgaria). - In: Proceedings of the Balkan Scientific Conference of Biology in Plovdiv (Bulgaria) frm $19^{\text {th }}$ till $21^{\text {st }}$ of May 2005, (Eds. B. Gruev, M. Nikolova, A. Donev), 428--435.

Georgiev D. \& Georgiev B., 2002: Terrestrial Gastropods as Intermediate Hosts of Protostrongylid Nematodes in Pastures for Sheep and Goats in the Region of Stara Zagora, Bulgaria. - Acta Zoologica Bulgarica, 54(3): 47-54.

Georgiev D. \& Georgiev B., 2003: Land snails in the transmission of protostrogylids on pastures in Southern Bulgaria: variability of infection levels related to environmental factors. - Acta Parasitologica, 48(3): 208-217.

Georgiev D. \& Georgiev B., 2004: Features of the Malacofauna on the pastures from the region of Stara Zagora and its importance for the circulation of the Protostrongylides. - Stockbreeding Sciences, XLI, 4: 88-92. (in Bulgarian).

Georgiev D. \& Stoycheva S., 2008: A record of Bythinella $\mathrm{cf}$. opaca (Gallenstein 1848) (Gastropoda: Prosobranchia: Hydrobiidae) in Bulgaria. - Malacologica Bohemoslovaca, 7: 51-54. Online serial at $<$ http://mollusca. sav.sk $>6$-Jun-2008.

Glöer P. \& Meier-Brook C., 2003: Süsswassermolluscen - Ein Bestimmungsschlüssel für die Bundesrepublik Deutschland. - Deutscher Jugendbund für Naturbeobachtung (Hrsg.), Hamburg, 13. neubearbeitete, Auflage, 134 pp.

Hausdorf B., 2000: The genus Monacha in Turkey (Gastropoda: Pulmonata: Hygromiidae). - Archiv für Molluscenkunde, 128 (1/2): 61-151.

Ноторр K., 2002: Land snails and soil calcium in central Appalachian Mountain forest. - Southeastern Naturalist, 1(1): 27-44. Hubenov Z., 2005: Malacofaunistic diversity of Bulgaria. - In: Current state of Bulgarian biodiversity - problems and perspectives, Petrova A. (ed.) Bulgarian Bioplatform, Sofia, 199-246. (in Bulgarian).

Kalisz P. \& Powell J., 2003: Effect of calcerous road dust on land snails (Gastropoda: Pulmonata) and millipedes (Diplopoda) in acid forest soils of the Daniel Boone National Forest of Kentucky, USA. - Forst ecology and management, 186: 177-183.

Kerney M., Cameron R. \& Jungbludgh J., 1983: Die Landschnecken Nord- und Mitteleuropas. - Verlag Paul Parey, Hamburg \& Berlin, $384 \mathrm{pp}$.

LožEK V., 1962: Soil conditions and their influence on terrestrial gasteropoda in Central Europe. - Program in Soil Zoology, London, 1: 334-342.

Schilthuizen M., Hsien-Nee C., Kimsin T, Vermuelen J., 2003: Abundance and diversity of land-snails (Mollusca: Gastropoda) on limestone hills in Borneo. - The Raffles Bulletin of Zoology, National University of Singapore, 51(1): 35-42.

WIKTOR A., 1983: The slugs of Bulgaria (Arionidae, Milacidae, Limacidae, Agriolimacidae - Gastropoda, Stylommatophora). - Annales Zoologici, 37 (3): 71-206. 